\title{
Negative Transfer of Liaoning Dialect on English Phonetics Learning
}

\author{
Jie Fu \\ College of Foreign Languages, Bohai University, Jinzhou, 121013, China \\ 563714244@qq.com
}

Keywords: Liaoning dialect; English phonetics learning; negative transfer; phonetics negative transfer; influence; strategies

\begin{abstract}
Dialect is a language variant that have its own characteristics in region or society. Dialect produce a great impact on students' English learning, not only affect pronunciation, can also affect the understanding of English syllables as well as the English intonation and weight control. Dialect's influence to English learning in English teaching and language acquisition is the key problems to be solved. In this paper, in order to reduce the negative transfer of Liaoning dialect and promote English learning and study. On the basis of the negative transfer of language transfer theory and phonetics negative transfer, this paper analyzed the characteristics of Liaoning dialect, pointed out the influence of dialect of English phonetics learning, puts forward the countermeasures for the English phonetics learning. In this paper, the research results, improve the learners' pronunciation accuracy in Liaoning plays an important role.
\end{abstract}

\section{Introduction}

Dialect is a variant of language, according to the nature of the dialect can be divided into regional dialects and social dialects. Regional dialect was due to the regional difference and form variation of language, language is the imbalance of regional development reflect; Social dialect are social members of the same area, because occupation, class, age, gender and cultural differences, and form the different social variations. Dialect even though only in certain areas, but it is also a complete system itself. Dialect has a voice structure system, the structure of the vocabulary and grammatical structure system, to meet the needs of the region's social communication. Dialect is a unique national culture, every place has a unique dialect, has a rich cultural background; Mandarin as the tool of interpersonal communication, popularization is important, dialect as a culture and art, contains a strong national characteristics, is a kind of social cultural phenomenon.

In English learning, accurate, fluent and idiomatic speech often can give people deep impression, this is show learners of English basic one of the most important aspects of the quality of language and communication skills. However, Chinese English learners in the process of learning English is affected by the Chinese pronunciation, the Chinese migration into the English learning pronunciation habits, has been plagued by phonetic learning, pronunciation is not standard, many problems of voice. Also, will also dialect pronunciation habits to English learning. Some English phonetics is not found in mandarin or dialect tone, will hinder the ability of English phonetics learning and mastering. In this paper, we study the Liaoning dialect influence on English phonetics learning, and to explore effective method of phonetic teaching.

\section{Language Transfer Theory}

"Transfer" is a psychological term, refers to learners in the learning process of the existing knowledge or skills to gain new knowledge or skills and influence. Language transfer is to point to in the second language learning, learners in the use of a second language, with the help of the native language rules of pronunciation, meaning, structure or used to express ideas. If the mother tongue language rules and foreign language is consistent, so the rules of the native language transfer would have positive effects on the target language, this is called positive transfer. Negative transfer refers to the language rule if the mother tongue is not in conformity with the foreign language habits, have negative effects on foreign language learning. Can explain language migration between different 
ethnic groups in the history of language contact and language change, theoretical foundation includes the following [1, 2]:

(1) Behaviorism theory. Learner language environment is crucial to language learning. Learners obtain language stimulation is correct, has it been possible to make the right response; Only the basic sentence patterns of a large number of the target language learners imitate, can eventually become a habit in the brain and internalization. Language learning should follow the law of first language acquisition, or learn to hear that you read and write. Reinforcement is an important factor in language learning.

(2) Contrast analysis theory. Comparing the two languages and determine the similarities and differences. Ultimate goal is to predict tongue on the possible impact of second language learning, namely the learner of a second language by mother tongue interference may appear error, so as to determine the emphasis and difficulties in teaching, the corresponding preventive measures. Part of the same mother tongue and target language most likely positive transfer effect, not completely the same mother tongue and target language but a part of the negative transfer may occur.

(3) Intermediary language theory. For the second language acquisition, the whole learning process with the mother tongue migration and the target language rules generalization, resulting in a series of gradual approach, but always different from the native language and the target language in the middle of the transition state of language, referred to as "interlanguage". The cause of interlanguage is divided into five aspects: language transfer, the generalization of the target language rules, training transfer, omitted to simplify, avoidance and change.

\section{Phonetics Negative Transfer}

In learning English, no matter how much Chinese students speak English fluently, have a bit of Chinese accent, and could not speak pure authentic like native speakers. The reason is that once the learners missed the critical period of learning English, learning English can't be like learning language can easily nature.

First of all, from the perspective of the pronunciation learning English is by lung airflow compression to send sound, feels more vigorous; While the Chinese general from oral pronunciation, feel soft. So, Chinese people speak English with foreigners than, give a person a kind of soft feeling, so as to bring foreign accent marks. This is a kind of common pronunciation characteristics of interlanguage fossilization, especially in high school. Second, from the speech language system, English is a kind of rely mainly on tone to distinguish meanings of "intonation language", and Chinese belongs to the tone differences between meanings of "tone language", the two languages in terms of the number of phonemes and their combination differs very big.

First of all, English not only have ends in a vowel syllable word, also have closed syllable word ends in a consonant sound, and Chinese word ends in a vowel sound. But students often after English consonant plus a vowel, so "work" is "the worker" by reading, "bet" read into "better".

Second, some English phonemes in Chinese doesn't work, so, "' thank, sing, shy" is often read as "[senk] [sin] [sai]", especially students dialect is very heavy. No English consonant clusters in Chinese, always separated by a vowel between Chinese consonants, thus, reading the English consonant clusters, students tend to add a vowel in the middle.

Third, the loss of blasting, the soft consonants in English, even read and stress, etc., also often make students puzzled, easy to cause the voice of the migration.

\section{Characteristics of Liaoning Dialect}

Liaoning province is located in the south of northeast China, the south near the Bohai sea, the yellow sea and echo of Shandong province; East and north Korea, and Japan, South Korea across the sea; Hebei province and Inner Mongolia west, north and adjacent to Jilin strategic position is very important. Is the only coastal and border provinces in northeast China, the eastern region is the northeast and Inner Mongolia autonomous region opening to the outside of the portal [3]. It's the important part of northeast China economic zone and the Bohai sea economic zone, meanwhile is 
the important channel of northeast China's foreign trade and international exchange. Liaoning province area of 15 square kilometers and a population of more than 4000, and the natural environment is superior. Is one of the national minority large population provinces, fifth in the national minority population absolute sequence, the proportion of ethnic minority is in 10th. The general picture of terrain is roughly three points "six mountains, one river, and little land". Roughly terrain from north to south, since the things on both sides of the inclined to central China; Mountains and hills respectively on the two sides, down to the central plains, horseshoe-shaped leaning to the Bohai sea.

With a long history and culture of Liaoning province, Chaoyang Niuhe Lianghongshan culture site, about 5000 years ago, from the goddess unearthed, rubble, the temple and the altar, jade pig dragon portrait painted and painted pottery and other important cultural relics come to the conclusion: there is a certain national rudiment of primitive civilization, marked the Liaoning region is one of the birthplace of the Chinese nation culture. From a historical perspective, Liaoning merged the dialect vocabulary in the history of Manchu and Mongolian languages such as some of the vocabulary, Liaoning south area has absorbed Japan and South Korea and other foreign words. Liaoning dialect the internal consistency of the strong, the difference is not big. On pronunciation, vocabulary, grammar, the structure characteristics of [4-7] as follows:

(1) Mandarin zero initial syllables words, mostly read "w" initials. Such as: wushu and "wu" and "five" five "pronunciation is the same.

(2) Mandarin "n" initials words, such as "male, warm, take", usually read "n" initials. Mandarin zero initial open call words, such as "e, e, ai" and so on, in addition to "qingan, anda, zhaozhou" read "n" initials; "mohe, huma, heihe" read "zero" initials outside; Around the other read the initials "zero" or "n" initials, some still can freely interchangeable.

(3) Mandarin "r" initials, most places is read, "zero" initials. Such as: the "ren" of the people is the same as the "yin" in silver, like "yang" and "rang" of humility, same warm "re" and the darkness "ye" is the same, the beef "rou" and "you" of child, the same, the software of "ruan" and "yuan" of far is the same, if the "ru" and "yu" carp were the same.

(4) Mandarin labial initials "b, p, m, f" split with rhymes "o", read "e" rhyme, such as "bō= (bē) ".

\section{Influence of Dialect on English Phonetics Learning}

American structuralists linguists divided the phonemes into two categories, namely the segmental phoneme and suprasegmental phoneme. suprasegmental phoneme roughly equivalent to a vowel and consonant, constitutes the basic aspects of pronunciation system; And suprasegmental phoneme generally include stress and pitch and juncture, used to modify the sound segment phoneme [8]. Accordingly, the English phonetic structure including segmental structure and suprasegmental structure), in the actual flow of the English language, sound structure and suprasegmental structure is together. Passage of the grammar structure is used to express the lexical structure, suprasegmental structure of the grammar structure is used to express syntactic structure. The influence of the dialect of English phonetics learning including sound and supersound two aspects:

(1) Sound period of speech errors. Vowels and consonant pronunciation errors include:

Vowel, including unit and double vowel sounds. Monophthong mainly the phonological errors, read a phoneme into another phoneme, embodied in: unit between mutual confusion, unit note there is no corresponding in dialect tend to substitute an easy phonemes, in dialect sounds instead of unit of similar. The double vowel is one of the most common errors of the English pronunciation. Embodied in: double vowel of the first component pronunciation is bad, replace each other between the double vowels, turn the dual vowels into three vowels.

Consonant pronunciation mistakes include lack of phoneme, phoneme and phoneme confusion three conditions. Phoneme deletion is the lack of swallowing the sound or pronunciation way, increase the phoneme is in after ending consonants and vowels, phoneme confusion belongs to phoneme swaps. Phoneme swap includes pronunciation way or pronunciation deviation. 
Pronunciation way is transferring, such as soft consonants and voiced consonants confusion; Place of articulation migration, such as nasal pronunciation deviation.

(2) Period of speech errors. Supersound segment is phonetics and sound system is to refer to the words of covering more than one tone of voice effect, speech errors can be divided into the following types:

Syllable structure caused by mistake. Differences between English and Chinese syllable structure led to the incorrect phenomenon of English pronunciation. Due to no consonant cluster structure in Chinese, there is always a vowel between consonants, and so when you meet the English consonant clusters often plugged into a vowel in the middle.

Stress types and distribution caused by mistake. Stress refers to a syllable. Stress error is generally characterized by read English sentences is very stiff, word stress is not prominent, stress or too much stress.

Language rhythm type caused by mistake. Rhythm is available to unstressed syllables, stressed syllables length or pitch combinations to illustrate. Common mistake is assigned to the length of the stressed and unstressed syllables.

Tone type caused by mistake. English and Chinese intonation types in different area all the students of English intonation lack of ups and downs, and use more falling tone, use some Chinese tone type to replace English adjustable type.

\section{Strategies for English Phonetics Learning}

In view of the negative transfer function of Liaoning dialect, through in-depth analysis of the impact of English phonetics learning dialects, absorb the research achievements of related literature [9], this paper put forward the countermeasures for the English phonetics learning as follows:

(1) Eliminate the learner's psychological effect. Language migration is influenced by factors such as learners' personality and teaching environment. If the student's psychological pressure is too large, it will directly affect the effect of English teaching. As much as possible in order to promote the positive transfer of language, teachers create the right environment, eliminating psychological barriers of students, help students analyze the cause of negative factors, causes the student to put down the burden, with confidence, and create conditions for promoting the positive transfer of language. Modern education emphasizes the concept of people-oriented, the student is the main body of teaching activity, plays a decisive role in the teaching. Many years of English teaching practice has proved, into the students' psychological, eliminate students' psychological barriers, grope for the students' English teaching style, to find the path to success in teaching English.

(2) Use the portfolio strategy in phonetic teaching. Portfolio strategy includes three aspects: one is, handle the relationship of the interpretation of the pronunciation rules and imitating practice. Teachers firstly to briefly explain rules and pronunciation, and then learner to imitate practice. We should give priority to with learners imitate, on the basis of correct imitation, a large number of practices. The second is, phonetic teaching and writing teaching. Phonetic teaching training should be related with writing skills. At the same time in the stressed the importance of pronunciation and intonation, cannot ignore writing training. Do hear, read ahead and timely keep up with the writing, listening, speaking, reading and writing. Three is, pay attention to cultivate learners' speech skill. To do fast blurt out correctly, the learner's pronunciation organs to adapt to a series of actions, avoid pronunciation by mother tongue interference.

(3) Exclude mother tongue interference to strengthen English thinking. To overcome the interference of mother tongue in English, to convert the Chinese thinking in English training is very necessary. Students in the process of speak or write in English, there has been no jump out of the native way of thinking. Group of sentence is not according to the English way of thinking, make sentences according to Chinese habit good organization, and then translated into English. The wrong way of thinking should be classified as a syntax error it is misunderstanding. Grammar is a surface phenomenon, the reason is that English language logic thinking is flawed, represented by mistakes in grammar. Learning to learn English in the process of thinking, ways include two aspects: one is that starting from imitation, learning a language is the main means of mimicry, starting from 
hearing orientation activities, through the brain analyzer, and the psychological activity organs exercise complete; Second, get rid of the native language, way of thinking in English to English.

(4) Language as the tool of passing information and communication. The main purpose of learning English is to use English for communication, including oral and written in two forms, therefore, teachers should attach importance to the output of the language, students in the process can realize their own language, fixed incorrect language habit; Teachers can also get feedback, recognize the student's stage of English learning, correctly treat students' errors, help students to find suitable learning strategies, which can be targeted to improve the students' English level. In addition, teachers should encourage students to more communication, organization all kinds of English extracurricular activities, to improve students' English pragmatic competence in the process of communication.

\section{Conclusion}

Phonetics transfer is the most prominent phenomenon of language transfer. Chinese and English pronunciation system are different, Chinese characters with four tones to distinguish different meaning, referred to as a tonal languages. English words don't tone distinguishes the righteousness, sentence by tone distinguishes the righteousness, called intonation language; Chinese characters generally don't have to end consonants, and English is no such rules; English and Chinese factors is not corresponding. In Liaoning dialect, it's difficult to separate some phonemes in pronunciation, so the pronunciation of the English learners. This article research results to reduce the negative transfer function of Liaoning dialect, promote English learning, to improve learners' pronunciation accuracy and so on is of great significance.

\section{Acknowledgment}

This work is supported by social science fund project of Liaoning province (L15BYY007): Phonetics Characteristics of Liaoning dialect and its influence on English education in Liaoning.

\section{References}

[1] C. H. Qin, "Exploring the language transfer in second language acquisition," Journal of Changsha Railway University (Social Science), vol. 15, no. 3, pp. 141-122, 2014.

[2] Y. Gao, "The study of language transfer in second language acquisition," Journal of the Socialism Institute of Yunnan, http://www.cnki.net/kcms/detail/53.1133.D.20140219.0036.OS l .html, 2014-02-19.

[3] G. L. OuYang, "Summary on the Research of Liaoning Dialect in Past 60 Years," Huazhong Normal University Journal of Postgraduates, vol. 17, no. 4, pp. 50-52, 2010.

[4] First constellation network, "Characteristics and division of Liaoning dialect," http://www.d1xz.net/wenhua/chengshi/art98021.aspx, 2016-5-9.

[5] X. M. Chen, "Phonological Studies on Liaoning Dialect," Journal of Liaoning Normal University (Social Science Edition), vol. 33, no. 5, pp. 93-99, 2010.

[6] N. Ding, "Liaoning dialect phonetic research overview," Journal of Jiamusi Vocational Institute, vol. 32, no. 11, pp. 322, 2015.

[7] L. B. Tang, "A review of the study of language transfer," Modern Chinese, vol. 12, no. 5, pp. 16-19, 2015.

[8] X. H. Li, "An analysis of English suprasegmental effects," Journal of Shengli Oilfield Teachers College, vol. 15, no. 2, pp. 48-50, 2001. 
[9] Q. Y. Zhang, "The influence of Liaoning Dialect on College Students' English learning," Crazy English (Teachers), vol. 4, no. 12, pp. 57-60, 2007.

[10] X. M. Li, "The Influence of Dialect on Pronunciation Acquisition of English Learners," Journal of Lanzhou Jiaotong University, vol. 34, no. 2, pp. 82-84, 2015. 